\title{
Physical Performance Correlates with Self-Reported Physical Function and Quality of Life in Patients at 3 Months after Total Knee Arthroplasty
}

\author{
Jun Hwan Choi ${ }^{1}$, Bo Ryun Kim², Sang Rim Kim ${ }^{3}$, Kwang Woo Nam ${ }^{3}$, So Young Lee ${ }^{1}$, Won Bin Kim ${ }^{1}$, Youn Ji Kim ${ }^{1}$ \\ ${ }^{1}$ Department of Rehabilitation Medicine, Regional Rheumatoid and Degenerative Arthritis Center, Jeju National University Hospital, Jeju National University \\ College of Medicine, Jeju, Korea \\ ${ }^{2}$ Department of Physical Medicine and Rehabilitation, Korea University Anam Hospital, Seoul, Korea \\ ${ }^{3}$ Department of Orthopedic Surgery, Regional Rheumatoid and Degenerative Arthritis Center, Jeju National University Hospital, Jeju National University College \\ of Medicine, Jeju, Korea
}

Corresponding Author:

Bo Ryun Kim, MD, $\mathrm{PhD}$

Department of Physical Medicine and

Rehabilitation, Korea University Anam

Hospital, 73 Goryeodae-ro, Seongbuk-

gu, Seoul 02841, Korea

E-mail: brkim08@gmail.com

ORCID:

https://orcid.org/0000-0001-7788-7904

Received: March 31, 2020

Revised: May 21, 2020

Accepted: May 21, 2020
Background: Although total knee arthroplasty (TKA) is an effective treatment for knee osteoarthritis, assessment of postoperative outcomes remains unclear. This study aimed to identify postoperative physical performance factors that are correlated with self-reported physical function and quality of life $(\mathrm{O} \mathrm{OL})$ at 3 months after unilateral TKA. Methods: In total, 158 patients who underwent unilateral primary TKA completed performance-based physical function tests at 3 months after surgery, including Stair Climbing Tests (SCT), 6-Minute Walk Tests (6MWT), Timed Up and Go tests (TUG), and instrumental gait analysis. We also measured the isometric knee flexor and extensor strengths of the operated and non-operated knees. Self-reported physical function and QoL were assessed using the Western Ontario and McMaster Universities Osteoarthritis Index (WOMAC) and the Euro-OoL Five Dimensions (EQ-5D) questionnaire, respectively. Results: Bivariate analyses showed that WOMAC function and EQ-5D were correlated with age, other self-reported measures, and performance-based measures. The WOMAC pain $(r=0.71, p<0.001)$ showed a high positive correlation. While the EQ-5D $(r=-0.7, p<0.001)$ showed a highly negative correlation with WOMAC function, WOMAC pain $(r=-0.67, p<0.001)$ showed a moderately negative correlation with EQ-5D. In multivariate linear regression analyses, WOMAC pain, peak torque of the flexor of the non-operated knee, and reductions in extensor and stride length were associated with self-reported physical function, whereas WOMAC pain, SCT ascent, and cadence were associated with postoperative QoL. Conclusions: Physical performance factors were significantly associated with self-reported physical function and $\mathrm{OoL}$ in patients at 3 months after unilateral TKA. These findings suggest that performance-based physical function could be used to assess outcomes after TKA.

Key Words: Osteoarthritis, Knee, Arthroplasty, Rehabilitation, Quality of life

\section{INTRODUCTION}

Knee osteoarthritis $(\mathrm{OA})$ is the single most common joint disease and a major cause of disability in older adults. Pain and swelling of the affected joints can reduce mobility and impair activities of daily living and quality of life (QoL).,2)
Total knee arthroplasty (TKA) is an effective and cost-efficient treatment for patients with end-stage knee arthritis. ${ }^{3)}$ The most prevalent preoperative expectations of TKA are relief of pain. ${ }^{4,5)}$ However, despite pain relief after successful TKA, patient expectations of physical function and QoL are often unfulfilled. ${ }^{6,7)}$

Physical function changes over time after TKA. Our previous 
study showed correlations between preoperative performance-based physical function and self-reported physical function and QoL. ${ }^{8)}$ Dynamic balance and exercise capacity were powerful predictors of self-reported physical function and QoL. During the first month after TKA, physical performance and the results of self-reported questionnaires worsen substantially from preoperative conditions. ${ }^{9,10)}$ Performance-based measures showed greater responsiveness compared to self-reported questionnaires early after surgery and patients tend to overestimate actual short- and long-term changes in physical function after TKA. ${ }^{11)}$ In addition, several studies using both questionnaires and physical performance functions have shown that the self-reported physical function of individuals often differs substantially from their actual functional capability. ${ }^{12,13)}$ Due to the discrepancies between self-reported and performance-based physical function, correlation analysis of both outcomes is necessary in patients who undergo unilateral TKA. By the third postoperative month, self-reported physical function and QoL scores usually surpass preoperative values. ${ }^{14)}$ Moreover, the greatest improvements in gait and lower extremity function occur during the first 3 months after TKA. ${ }^{15)}$ By this time, most patients have resumed their daily activities in the community and at home.

We previously reported improvement in functional outcomes during the first 3 months after the application of a critical pathway for patients who underwent TKA. ${ }^{16)}$ This study included patients who underwent unilateral and bilateral TKA; however, we did not assess the relationship between physical performance and self-reported physical function.

Identifying performance-based measures that affect self-reported measures will likely be meaningful; moreover, it is important to identify factors that prevent patients from fulling their expectations after TKA. Therefore, this study aimed to identify postoperative physical performance factors related with self-reported physical function and QoL at 3 months after unilateral TKA. The results of this study may guide postoperative rehabilitation strategies to improve physical performance and self-reported physical function and QoL.

\section{MATERIALS AND METHODS}

\section{Study Design and Participants}

This retrospective cross-sectional study enrolled 158 patients who were diagnosed with knee $\mathrm{OA}$ and underwent unilateral primary TKA between October 2013 and May 2019. The inclusion criterion for this study was patients who walk independently with or without an ambulatory aid after surgery. Patients with previous neurological, cardiorespiratory, or orthopedic disease that caused ambulatory deficits were excluded.
All patients completed performance-based physical function tests, including Stair Climbing Tests (SCTs), 6-Minute Walk Tests (6MWTs), Timed Up and Go tests (TUGs), and instrumental gait analyses. We also measured the isometric knee flexor and extensor strengths of the operated and non-operated knees. Self-reported physical function and pain were measured using the Western Ontario and McMaster Universities Osteoarthritis Index (WOMAC), and self-reported QoL was measured using the Euro-QoL Five Dimensions (EQ-5D) questionnaire.

We informed the patients about the nature of the study and its risks and benefits and all participants provided written consent. The study protocol was approved by the Institutional Review Board at Jeju National University Hospital (No. JEJUNUH 201912-013).

\section{Rehabilitation Protocol}

Beginning on the first day after TKA, patients underwent a standard rehabilitation program including passive knee range of motion (ROM) and physical modalities such as cryotherapy and transcutaneous electrical nerve stimulation for relief of knee pain and swelling. The patients started progressive resistance strengthening exercises 7 days after surgery and an intensive rehabilitation program including gait training, aerobic exercises using an ergometer, functional training for transfer, and stair climbing beginning on postoperative day 14 . The patients participated in the rehabilitation programs twice daily, five times per week for 2 weeks in the rehabilitation department under the supervision of physical therapists.

\section{Outcome Measures}

All patients underwent assessments 3 months after surgery, with physical performance and self-reported physical function and QoL tests performed on the same day.

\section{Assessments of performance-based physical function}

6MWT: The 6MWT is a performance-based measure of functional exercise capacity, such as walking capacity and gait endurance, in adults. In this test, the participants walk as far as possible for 6 minutes along a 50-m hallway marked with lines. ${ }^{17)}$

TUG: The TUG test is used to evaluate a dynamic balance. In this test, the participants sit with their backs against a chair (seat height, $44 \mathrm{~cm}$; width, $49 \mathrm{~cm}$; armrest height, $64 \mathrm{~cm}$ ) placed at the end of a marked 3-m distance and stand up upon hearing the word "go", walk at a comfortable speed past the 3-m mark, turn around, walk back, and sit down again in the chair without physical assistance, all while being timed. ${ }^{18)}$

SCT: The SCT is a measurement of the time required to ascend 
and descend a flight of 12 steps, each $17 \mathrm{~cm}$ high and $25 \mathrm{~cm}$ wide. In this test, the participants ascend or descend the stairs as fast as possible upon hearing the word "go". Each patient completed three trials, with a 5-minute rest interval between each pair of trials. The fastest time was recorded for each patient. ${ }^{19)}$

Measurement of knee ROM: The ROM of the affected knee was measured using a standard long-arm goniometer. The axis, movement arm, and stationary arm of the goniometer were aligned with the center of the lateral epicondyle of the femur, the lateral malleolus, and the greater trochanter of the femur, respectively. The knee flexion ROM was measured as the maximal active bending of the knee with the patient in the supine position. Knee extension ROM was measured as the angle of maximal active straightening with the patient's heel propped on a $10-\mathrm{cm}$ wooden block. The degree of extension beyond zero for hyperextension during this assessment was recorded as a negative value. ${ }^{20,21)}$

Gait analysis: A wireless inertial sensing device (G-WALK; BTS Bioengineering S.p.A., Milan, Italy) was used to measure the spatiotemporal variables of gait. The patients wore a semi-elastic back-belt device on the waist to measure the acceleration of the anteroposterior, mediolateral, and vertical axes and were instructed to walk barefoot along an 8-m pathway at a comfortable speed. Gait data were transmitted via Bluetooth to a personal computer and were processed using the BTS G-WALK system, a dedicated software program that measures length, duration, and single supports to calculate a typical gait curve. The second positive peak on the curve was the instant at which the patient's foot contacted the ground. Therefore, the step length was defined as the distance between two successive foot contacts with the ground, while stride length was defined as the distance between three successive foot contacts. The first and last steps were removed from all calculations because of abnormal patterns and psychological responses at the start and end of walking, respectively. ${ }^{22)}$

Measurements of isometric strengths of knee extensors and flexors: A physical therapist used an isokinetic dynamometer (HUMAC NORM; Computer Sports Medicine Inc., Stoughton, MA, USA) to measure the maximal isometric strengths of the bilateral knee extensors and flexors. All patients were instructed to start a structured warm-up with the knee joint fixed at $60^{\circ}$ of flexion to generate maximal isometric force, followed by maximal voluntary contractions until the torque did not increase by more than $5 \%$ during three successive attempts. The patients then performed knee flexion and extension as discrete movements in a single direction. Each contraction lasted 4-5 seconds, with 2-minute rest intervals between contractions. After a 5-minute rest, the patients repeated the procedure on the other lower limb. The variables measured included the peak torques (PTs) of the extensor and flexor, the ratio of hamstring to quadriceps strength ( $\mathrm{H} / \mathrm{Q}$ ratio), and the difference in the strengths of the extensors and flexors between the operated and non-operated knees, expressed as percentages of the strengths of the non-operated knee. ${ }^{21,23)}$

\section{Assessment of self-reported physical function and $Q o L$}

WOMAC: The multidimensional WOMAC index is a questionnaire used to assess pain, stiffness, and physical functional disability $^{24)}$ and has previously been applied to measure self-reported disability in patients with knee OA. The WOMAC index comprises 24 variables: 5 addressing pain, 2 addressing stiffness, and 17 addressing physical function. Each of these variables is scored using a 5 -point Likert scale ( $0=$ none, $1=$ slight, $2=$ moderate, 3 =very, $4=$ extremely). The WOMAC index measures the degree of pain, stiffness, and difficulty in performing 17 activities during the preceding 48 hours. Higher scores indicate greater levels of pain, stiffness, and difficulty.

EQ-5D questionnaires: The EQ-5D questionnaire is widely used to evaluate self-reported QoL by measuring five dimensions of QoL: mobility, self-care, usual activities, pain/discomfort, and anxiety/depression. ${ }^{25)}$ Each dimension has three levels of severity (no problems, some or moderate problems, and extreme problems). The scores were converted using utility weights derived from the general Korean population and ranged from -1 to 1. Lower scores indicated worse overall health status.

\section{Statistical Analysis}

All variables were subjected to descriptive statistics. We used Pearson correlation analysis to assess the relationships between postoperative self-reported physical function and QoL, and physical performance. Multivariate linear regression analysis using a backward selection linear regression model was used to determine the postoperative physical performance factors independently associated with self-reported physical function and QoL 3 months after TKA. We used SPSS for Windows version 20.0 (IBM SPSS, Armonk, NY, USA) to perform all analyses, with $\mathrm{p}<0.05$ considered statistically significant.

\section{RESULTS}

This study enrolled 158 patients ( 134 women and 24 men) with a mean age of $72.6 \pm 5.8$ years and mean body mass index (BMI) of $25.6 \pm 3.0 \mathrm{~kg} / \mathrm{m}^{2}$. Their baseline demographic and disease-related characteristics are presented in Table 1 . Of these 158 patients, 131 (82.9\%) had Kellgren-Lawrence grade IV knee OA. 
Table 1. Demographic and disease-related characteristics of the subjects $(\mathrm{n}=158)$

\begin{tabular}{lc}
\hline Characteristic & Value \\
\hline Age $(y)$ & $72.6 \pm 5.8$ \\
Sex & \\
$\quad$ Male & $24(15.2)$ \\
Female & $134(84.8)$ \\
BMI $\left(\mathrm{kg} / \mathrm{m}^{2}\right)$ & $25.6 \pm 3.0$ \\
K-L grade & \\
3 & $27(17.1)$ \\
4 & $131(82.9)$ \\
Comorbidities & \\
Osteoporosis & $81(51.3)$ \\
Pre-sarcopenia & $7(4.4)$ \\
Degenerative spine disease & $26(16.5)$ \\
Diabetes mellitus & $29(18.4)$ \\
Hypertension & $106(67.1)$ \\
\hline
\end{tabular}

Values are presented as mean \pm standard deviation or number (\%). BMI, body mass index; K-L grade, Kellgren-Lawrence grade.

\section{Postoperative Evaluation of Performance-based Physical function, Self-reported Physical Function, and QoL and Their Correlations}

Table 2 presents the average postoperative performance-based physical function, self-reported physical function, and QoL scores of these patients.

In bivariate analyses, WOMAC function showed significant positive correlations with age $(\mathrm{r}=0.29, \mathrm{p}<0.001)$, WOMAC pain score $(r=0.71, p<0.001)$, WOMAC stiffness score $(r=0.24$, $p=0.003)$, TUG $(r=0.22, p=0.005)$, SCT ascent $(r=0.24$, $\mathrm{p}=0.003)$, SCT descent $(\mathrm{r}=0.22, \mathrm{p}=0.007)$, and knee extensor deficit $(r=0.16, p=0.047)$. WOMAC function also showed significant negative correlations with EQ-5D score $(r=-0.70, p<0.001)$, stride length $(r=-0.19, r=0.022)$, and PT of the flexor of the operated $(r=-0.38, p<0.001)$ and non-operated $(r=-0.47, p<0.001)$ knees.

EQ-5D scores showed significant negative correlations with age $(\mathrm{r}=-0.30, \mathrm{p}<0.001)$, WOMAC pain score $(\mathrm{r}=-0.67, \mathrm{p}<0.001)$, WOMAC stiffness score $(r=-0.16, p=0.043)$, WOMAC function score $(r=-0.70, p<0.001)$, TUG $(r=0.22, p=0.005)$, SCT ascent $(\mathrm{r}=0.24, \mathrm{p}=0.003)$, and SCT descent $(\mathrm{r}=0.22, \mathrm{p}=0.007)$. EQ$5 \mathrm{D}$ scores also showed significant positive correlations with $6 \mathrm{MWT}(\mathrm{r}=0.31, \mathrm{p}<0.001)$, cadence $(\mathrm{r}=0.22, \mathrm{p}=0.007)$, and the $\mathrm{PT}$ of the flexors of the operated $(\mathrm{r}=0.24, \mathrm{p}=0.002)$ and non-operated $(r=0.27, p=0.001)$ knees (Table 3).
Table 2. Postoperative evaluation of performance-based physical function, self-reported physical function, and quality of life in patients 3 months after unilateral TKA

\begin{tabular}{lc}
\hline Variable & Value \\
\hline 6MWT (m) & $443.41 \pm 101.76$ \\
TUG (sec) & $9.24 \pm 1.78$ \\
SCT (sec) & \\
Ascent & $10.74 \pm 3.94$ \\
Descent & $12.01 \pm 3.96$ \\
ROM (o) & \\
Affected ROM flexion & $124.66 \pm 11.59$ \\
Affected ROM extension & $-7.69 \pm 5.43$ \\
Gait linear parameters & \\
Gait speed (m/s) & $1.20 \pm 0.82$ \\
Cadence (steps/min) & $120.09 \pm 12.97$ \\
Stride length (cm) & $111.75 \pm 20.71$ \\
Gait cycle duration (sec) & $1.66 \pm 8.07$ \\
Stance phase duration (\% of gait cycle) & $64.67 \pm 3.39$ \\
Swing phase duration (\% of gait cycle) & $35.57 \pm 1.81$ \\
Double support duration (\% of gait cycle) & $27.91 \pm 4.82$ \\
Single support duration (\% of gait cycle) & $35.57 \pm 3.21$ \\
Isometric strength test & \\
PT of the extensor of the operated knee (Nm) & $80.47 \pm 26.00$ \\
PT of the extensor of the non-operated knee (Nm) & $102.92 \pm 63.65$ \\
PT of the flexor of the operated knee (Nm) & $51.95 \pm 14.05$ \\
PT of the flexor of the non-operated knee (Nm) & $52.56 \pm 15.26$ \\
Extensor deficit (\%) & $21.56 \pm 21.02$ \\
Flexor deficit (\%) & $10.09 \pm 14.86$ \\
Self-reported physical function & \\
WOMAC-Pain & \\
WOMAC-Stiffness & \\
WOMAC-Function & \\
Self-reported quality of life & \\
EQ-5D & \\
\hline
\end{tabular}

Values are presented as mean \pm standard deviation or number (\%).

TKA, total knee arthroplasty; WOMAC, Western Ontario and McMaster Universities Osteoarthritis Index; 6MWT, 6-Minute Walk Test; TUG, Timed Up and Go test; SCT, Stair Climbing Test; EQ-5D, Euro-QOL Five Dimensions; ROM, range of motion; PT, peak torque.

\section{Factors related with Self-reported Physical Function and QOL by Multivariate Linear Regression Analysis}

Postoperative WOMAC-Function score was significantly and independently associated with age $(\beta=0.15, p=0.011)$, WOMAC pain score $(\beta=0.59, \mathrm{p}<0.001)$, stride length $(\beta=-0.15, \mathrm{p}=0.009)$, $\mathrm{PT}$ of the flexor of the non-operated knee $(\beta=-0.31, \mathrm{p}<0.001)$, and extensor deficit $(\beta=0.16, p=0.006)$. The postoperative EQ$5 \mathrm{D}$ score was significantly and independently associated with the 
Table 3. Correlations between postoperative self-reported physical function and quality of life and physical performance in patients 3 months after unilateral TKA

\begin{tabular}{|c|c|c|}
\hline \multirow[b]{2}{*}{ Variable } & \multicolumn{2}{|c|}{ Correlation coefficients $(\mathrm{r})$} \\
\hline & $\begin{array}{l}\text { WOMAC } \\
\text { function }\end{array}$ & EQ-5D \\
\hline Age (y) & $0.29^{*}$ & $-0.30^{*}$ \\
\hline $\mathrm{BMI}\left(\mathrm{kg} / \mathrm{m}^{2}\right)$ & -0.08 & -0.01 \\
\hline \multicolumn{3}{|l|}{ Self-reported physical function } \\
\hline WOMAC-Pain & $0.71^{*}$ & $-0.67^{*}$ \\
\hline WOMAC-Stiffness & $0.24^{*}$ & $-0.16^{*}$ \\
\hline WOMAC-Function & 1 & $-0.70^{*}$ \\
\hline \multicolumn{3}{|l|}{ Self-reported quality of life } \\
\hline EQ-5D & $-0.70^{*}$ & 1 \\
\hline 6MWT (m) & 0.01 & $0.31^{*}$ \\
\hline TUG (sec) & $0.22^{*}$ & $-0.42^{*}$ \\
\hline \multicolumn{3}{|l|}{ SCT $(\mathrm{sec})$} \\
\hline Ascent & $0.24^{*}$ & $-0.41^{*}$ \\
\hline Descent & $0.22^{*}$ & $-0.43^{*}$ \\
\hline \multicolumn{3}{|l|}{$\mathrm{ROM}(\mathrm{o})$} \\
\hline Affected ROM flexion & 0.10 & -0.07 \\
\hline Affected ROM extension & -0.14 & 0.14 \\
\hline \multicolumn{3}{|l|}{ Gait linear parameters } \\
\hline Gait speed (m/s) & -0.02 & -0.03 \\
\hline Cadence (steps/min) & -0.03 & $0.22^{*}$ \\
\hline Stride length $(\mathrm{cm})$ & $-0.19^{*}$ & 0.10 \\
\hline Gait cycle duration (sec) & -0.04 & 0.04 \\
\hline Stance phase duration (\% of gait cycle) & 0.00 & -0.10 \\
\hline Swing phase duration (\% of gait cycle) & 0.12 & -0.06 \\
\hline Double support duration (\% of gait cycle) & 0.11 & -0.02 \\
\hline Single support duration (\% of gait cycle) & 0.02 & -0.09 \\
\hline \multicolumn{3}{|l|}{ Isometric strength test } \\
\hline PT of the extensor of the operated knee (Nm) & -0.15 & 0.14 \\
\hline PT of the extensor of the non-operated knee (Nm) & -0.13 & 0.11 \\
\hline PT of the flexor of the operated knee (Nm) & $-0.38^{*}$ & $0.24^{*}$ \\
\hline PT of the flexor of the non-operated knee (Nm) & $-0.47^{*}$ & $0.27^{*}$ \\
\hline Extensor deficit (\%) & $0.16^{*}$ & -0.08 \\
\hline Flexor deficit (\%) & 0.02 & 0.13 \\
\hline
\end{tabular}

TKA, total knee arthroplasty; BMI, body mass index; WOMAC, Western Ontario and McMaster Universities Osteoarthritis Index; 6MWT, 6-Minute Walk Test; TUG, Timed Up and Go test; SCT, Stair Climbing Test; EQ-5D, Euro-QOL Five Dimensions; ROM, range of motion; PT, peak torque. ${ }^{*} \mathrm{p}<0.05$.

WOMAC pain score $(\beta=-0.62, \mathrm{p}<0.001)$, SCT ascent $(\beta=-0.18$, $\mathrm{p}=0.013)$, and cadence $(\beta=0.13, \mathrm{p}=0.031)$ (Table 4$)$.

\section{DISCUSSION}

The results of the present study showed that objective performance-based physical function was correlated with self-reported physical function and QoL at 3 months after unilateral TKA. WO-
MAC function and EQ-5D scores were correlated with age, other self-reported measures, and performance-based measures. Based on the classification of correlations as very high, high, moderate, low, or negligible, ${ }^{26)}$ the WOMAC pain score showed a highly positive correlation with the WOMAC function score $(\mathrm{r}=0.71$, $\mathrm{p}<0.001$, whereas the EQ-5D showed a highly negative correlation with WOMAC function score $(\mathrm{r}=-0.70, \mathrm{p}<0.001)$. Pain after TKA was inversely correlated with time to recovery ${ }^{27)}$ and preoperative pain is correlated with self-reported physical function. ${ }^{8)} \mathrm{Pa}-$ tients experience pain for up to 3 months after surgery; however, even reduced pain can negatively affect physical function. Our results suggested that preoperative and postoperative pain control in patients could improve their physical function.

Among the performance-based physical function parameters tested in the present study, strengthening of non-operated knee flexor and extensor had significant correlations with WOMAC function. Quadriceps strength is frequently reduced after TKA and may affect physical functions. ${ }^{10,29,30)}$ Reduced quadriceps strength after TKA was related to lower gait speed, ${ }^{31)}$ and mobility limitations have been reported to be associated with large deficits of strength in surgical knee. ${ }^{19)}$ Moreover, the ratio of quadriceps to hamstring muscle strength was a strong predictor of weight-bearing asymmetry. ${ }^{32)}$ Hamstring muscles on both the operated and non-operated sides were found to be weaker after unilateral TKA. Many rehabilitation programs, however, target quadriceps strengthening of operated side alone, resulting in less information regarding hamstring muscle strength. These results may explain our finding that PT flexor of the non-operated knee and a deficit of the extensor correlated negatively with WOMAC function, and may be useful predictors of postoperative self-reported physical function.

WOMAC-Function score is inversely correlated with gait speed and stride length. ${ }^{33)}$ Older adults with gait speed $<1 \mathrm{~m} / \mathrm{s}$ are at high risk of poor health-related outcomes. ${ }^{34)}$ Comfortable gait speeds for healthy women and men aged 70-79 years are 1.13 and $1.26 \mathrm{~m} / \mathrm{s}$, respectively. ${ }^{35)}$ The mean gait speed in our patients was $>1 \mathrm{~m} / \mathrm{s}$, which was comparable to that of healthy adults, suggesting that gait speed had little effect on their physical functions. Normal adults have a mean stride length of $1.39 \mathrm{~m},{ }^{36)}$ about $20 \%$ higher than that in our patient population, suggesting that stride length rather than gait speed might have affected their physical function. Together, these results may explain the negative correlation between stride length and WOMAC function. Rehabilitation strategies after TKA should emphasize pain relief, strengthening the knee extensor and flexor, and gait training to increase stride length to improve functional outcome.

We also found that EQ-5D scores at 3 months after unilateral 
Table 4. Factors associated with self-reported physical function and quality of life by multivariate linear regression analysis

\begin{tabular}{|c|c|c|c|}
\hline Outcome/independent factors & Standardized $(\beta)$ & p-value & Adjusted $\mathrm{R}^{2}$ \\
\hline Postoperative WOMAC-Function & & & 0.64 \\
\hline Age & 0.15 & 0.011 & \\
\hline WOMAC-Pain & 0.59 & $<0.001$ & \\
\hline Stride length $(\mathrm{cm})$ & -0.15 & 0.009 & \\
\hline PT of the flexor of the non-operated knee (Nm) & -0.31 & $<0.001$ & \\
\hline Extensor deficit (\%) & 0.16 & 0.006 & \\
\hline Postoperative EQ-5D & & & 0.58 \\
\hline Age & -0.18 & 0.004 & \\
\hline WOMAC-Pain & -0.62 & $<0.001$ & \\
\hline SCT ascent & -0.18 & 0.013 & \\
\hline Cadence (steps/min) & 0.13 & 0.031 & \\
\hline
\end{tabular}

The logistic regression analyses were adjusted for age, sex, and body mass index (BMI).

WOMAC, Western Ontario and McMaster Universities Osteoarthritis Index; PT, peak torque; EQ-5D, Euro-QOL Five Dimensions; SCT, Stair Climbing Test.

TKA were correlated with age, WOMAC pain, WOMAC stiffness, WOMAC function, 6MWT, TUG, SCT ascent, SCT descent, affected ROM extension, cadence, and PT flexor of the operated and non-operated knees. Among these performance-based physical function parameters. Lower pain scores and higher satisfaction scores have been reported in patients after TKA. ${ }^{27)}$ Our results suggested that postoperative pain control and improved physical performance could increase postoperative QoL.

WOMAC pain was strongly and significantly associated with postoperative EQ-5D. The goals of TKA are to reduce pain, restore knee mobility and function, and improve QoL in cases of severe knee OA. Our previous study also showed a correlation between preoperative pain and self-reported QoL. ${ }^{8)}$ Moreover, the most significant predictor of patient dissatisfaction is persistent pain after surgery. ${ }^{37)}$ The results of these and the current study showed that patients continued to experience pain, albeit reduced, for up to 3 months after TKA, which possibly affected their QoL. The patients in our study had relatively lower WOMAC pain scores than those in other studies. ${ }^{38)}$ These lower scores may have been due to the cross-sectional nature of this study, with all patients receiving adequate rehabilitation for 3 weeks. Nevertheless, our findings suggested that pain relief, even if the surgery was successful, is required to improve patient $\mathrm{QoL}$.

We also found that SCT ascent and cadence were useful factors of postoperative EQ-5D scores. Stair activity is one of three recommended performance-based measures in patients with knee OA. ${ }^{39)}$ Stair activity is frequently limited in these patients and, as well as being a goal of postoperative rehabilitation, necessary for patient safety and independence. The SCT test is the most responsive performance-based measurement of recovery early after TKA. ${ }^{11)}$

Reduced cadence leads to reduced gait speed after TKA. ${ }^{40,41)}$
Limited gait speed is a significant issue; thus, increasing gait speed is a rehabilitation goal in patients with knee OA. ${ }^{32)}$ However, because the gait speed of our patients was similar to the comfortable gait speed of healthy individuals, gait speed was unlikely to have a major effect on QoL 3 months after surgery. Thus, rehabilitation strategies should emphasize pain relief, stair activity, and gait training with increased cadence to improve QoL.

Although both gait function (including spatiotemporal parameters) and physical function (including lower limb strengthening) improve greatly during the first 3 months after TKA, patients' subjective expectations of physical function remain unfulfilled until 1 year after TKA. ${ }^{6}$ These parameters, as well as isometric strengthening of the knee 1 month after TKA, showed greater improvement in our previous study than in the present study. ${ }^{42)}$ Accordingly, the self-reported physical function and QoL 3 months after TKA may have been lower than the actually measured values.

Knee flexion and extension ROM at 3 months after TKA were not significantly correlated with physical function or QoL. Moreover, knee ROM at 1 month after TKA did not significantly affect physical function. These findings indicate that for at least 1 month after TKA, knee ROM is unrelated to physical function and QoL.

This study has some limitations. First, the results may not be generalizable to all TKA surgeries since the participants underwent only unilateral primary TKA. Accordingly, studies of patients who underwent bilateral TKA or revision are warranted. Second, the cross-sectional design of this study prevented analyses of the causal relationships among variables. Finally, we did not compare participants to those who did not receive postoperative rehabilitation after unilateral TKA.

In conclusion, the results of this study identified physical performance factors correlated with self-reported physical function and 
QoL in patients at 3 months after unilateral TKA. These findings suggest that these performance-based physical function could be used to assess outcomes after TKA.

\section{ACKNOWLEDGMENTS}

\section{CONFLICT OF INTEREST}

The researchers claim no conflicts of interest.

\section{AUTHOR CONTRIBUTION}

Conceptualization, BRK, JHC; Data curation, BRK, JHC, SYL, WBK, YJK; Investigation, BRK, JHC; Methodology: BRK, JHC; Project administration, BRK, JHC, SRK, KWN, SYL, WBK, YJK; Writing original draft, BRK, JHC; Review \& editing: BRK, JHC.

\section{REFERENCES}

1. Peat G, McCarney R, Croft P. Knee pain and osteoarthritis in older adults: a review of community burden and current use of primary health care. Ann Rheum Dis 2001;60:91-7.

2. Vos T, Flaxman AD, Naghavi M, Lozano R, Michaud C, Ezzati $\mathrm{M}$, et al. Years lived with disability (YLDs) for 1160 sequelae of 289 diseases and injuries 1990-2010: a systematic analysis for the Global Burden of Disease Study 2010. Lancet 2012;380:216396.

3. Cross WW 3rd, Saleh KJ, Wilt TJ, Kane RL. Agreement about indications for total knee arthroplasty. Clin Orthop Relat Res 2006;446:34-9.

4. Yoo JH, Chang CB, Kang YG, Kim SJ, Seong SC, Kim TK. Patient expectations of total knee replacement and their association with sociodemographic factors and functional status. J Bone Joint Surg Br 2011;93:337-44.

5. Mancuso CA, Sculco TP, Wickiewicz TL, Jones EC, Robbins L, Warren RF, et al. Patients' expectations of knee surgery. J Bone Joint Surg Am 2001;83:1005-12.

6. Nilsdotter AK, Toksvig-Larsen S, Roos EM. Knee arthroplasty: are patients' expectations fulfilled?: a prospective study of pain and function in 102 patients with 5-year follow-up. Acta Orthop 2009;80:55-61.

7. Jones CA, Voaklander DC, Johnston DW, Suarez-Almazor ME. Health related quality of life outcomes after total hip and knee arthroplasties in a community based population. J Rheumatol 2000;27:1745-52.

8. Hyun CW, Kim BR, Han EY, Kim SR. Preoperative physical performance predictors of self-reported physical function and quality of life in patients scheduled for total knee arthroplasty. J Phys Ther Sci 2016;28:3220-6.
9. Fitzgerald JD, Orav EJ, Lee TH, Marcantonio ER, Poss R, Goldman L, et al. Patient quality of life during the 12 months following joint replacement surgery. Arthritis Rheum 2004;51:100-9.

10.Mizner RL, Petterson SC, Snyder-Mackler L. Quadriceps strength and the time course of functional recovery after total knee arthroplasty. J Orthop Sports Phys Ther 2005;35:424-36.

11. Mizner RL, Petterson SC, Clements KE, Zeni JA Jr, Irrgang JJ, Snyder-Mackler L. Measuring functional improvement after total knee arthroplasty requires both performance-based and patient-report assessments: a longitudinal analysis of outcomes. J Arthroplasty 2011;26:728-37.

12. Wittink H, Rogers W, Sukiennik A, Carr DB. Physical functioning: self-report and performance measures are related but distinct. Spine (Phila Pa 1976) 2003;28:2407-13.

13. Hidding A, van Santen M, De Klerk E, Gielen X, Boers M, Geenen $\mathrm{R}$, et al. Comparison between self-report measures and clinical observations of functional disability in ankylosing spondylitis, rheumatoid arthritis and fibromyalgia. J Rheumatol 1994;21: 818-23.

14. Ethgen O, Bruyere O, Richy F, Dardennes C, Reginster JY. Health-related quality of life in total hip and total knee arthroplasty: a qualitative and systematic review of the literature. J Bone Joint Surg Am 2004;86:963-74.

15. Kennedy DM, Stratford PW, Riddle DL, Hanna SE, Gollish JD. Assessing recovery and establishing prognosis following total knee arthroplasty. Phys Ther 2008;88:22-32.

16. Kim JH, Kim BR, Kim SR, Han EY, Nam KW, Lee SY, et al. Functional outcomes after critical pathway for inpatient rehabilitation of total knee arthroplasty. Ann Rehabil Med 2019;43:65061.

17. Enright PL, McBurnie MA, Bittner V, Tracy RP, McNamara R, Arnold A, et al. The 6-min walk test: a quick measure of functional status in elderly adults. Chest 2003;123:387-98.

18. Podsiadlo D, Richardson S. The timed "Up \& Go": a test of basic functional mobility for frail elderly persons. J Am Geriatr Soc 1991;39:142-8.

19. Almeida GJ, Schroeder CA, Gil AB, Fitzgerald GK, Piva SR. Interrater reliability and validity of the stair ascend/descend test in subjects with total knee arthroplasty. Arch Phys Med Rehabil 2010;91:932-8.

20. Cibere J, Bellamy N, Thorne A, Esdaile JM, McGorm KJ, Chalmers A, et al. Reliability of the knee examination in osteoarthritis: effect of standardization. Arthritis Rheum 2004;50:45868.

21. Lee SY, Kim BR, Kim SR, Han EY, Nam KW, Park YG. Influence of preoperative physical function on gait 1 month after total knee arthroplasty. Ann Geriatr Med Res 2017;21:188-96. 
22. Bugane F, Benedetti MG, Casadio G, Attala S, Biagi F, Manca M, et al. Estimation of spatial-temporal gait parameters in level walking based on a single accelerometer: validation on normal subjects by standard gait analysis. Comput Methods Programs Biomed 2012;108:129-37.

23. Krishnan C, Williams GN. Variability in antagonist muscle activity and peak torque during isometric knee strength testing. Iowa Orthop J 2009;29:149-58.

24. Stucki G, Meier D, Stucki S, Michel BA, Tyndall AG, Dick W, et al. Evaluation of a German version of WOMAC (Western Ontario and McMaster Universities) Arthrosis Index. Z Rheumatol 1996;55:40-9.

25. Kim MH, Cho YS, Uhm WS, Kim S, Bae SC. Cross-cultural adaptation and validation of the Korean version of the EQ-5D in patients with rheumatic diseases. Qual Life Res 2005;14:1401-6.

26.Mukaka MM. A guide to appropriate use of correlation coefficient in medical research. Malawi Med J 2012;24:69-71.

27. Lamplot JD, Wagner ER, Manning DW. Multimodal pain management in total knee arthroplasty: a prospective randomized controlled trial. J Arthroplasty 2014;29:329-34.

28. Wailoo A, Hernandez Alava M, Escobar Martinez A. Modelling the relationship between the WOMAC Osteoarthritis Index and EQ-5D. Health Qual Life Outcomes 2014;12:37.

29. Berth A, Urbach D, Awiszus F. Improvement of voluntary quadriceps muscle activation after total knee arthroplasty. Arch Phys Med Rehabil 2002;83:1432-6.

30. Stevens JE, Mizner RL, Snyder-Mackler L. Quadriceps strength and volitional activation before and after total knee arthroplasty for osteoarthritis. J Orthop Res 2003;21:775-9.

31. Christiansen CL, Bade MJ, Weitzenkamp DA, Stevens-Lapsley JE. Factors predicting weight-bearing asymmetry 1 month after unilateral total knee arthroplasty: a cross-sectional study. Gait Posture 2013;37:363-7.

32. Pua YH, Seah FJ, Clark RA, Lian-Li Poon C, Tan JW, Chong HC. Factors associated with gait speed recovery after total knee arthroplasty: a longitudinal study. Semin Arthritis Rheum 2017; 46:544-51.

33. Nebel MB, Sims EL, Keefe FJ, Kraus VB, Guilak F, Caldwell DS, et al. The relationship of self-reported pain and functional impairment to gait mechanics in overweight and obese persons with knee osteoarthritis. Arch Phys Med Rehabil 2009;90:18749.

34. Cesari M, Kritchevsky SB, Penninx BW, Nicklas BJ, Simonsick EM, Newman AB, et al. Prognostic value of usual gait speed in well-functioning older people: results from the Health, Aging and Body Composition Study. J Am Geriatr Soc 2005;53:167580.

35. Bohannon RW, Williams Andrews A. Normal walking speed: a descriptive meta-analysis. Physiotherapy 2011;97:182-9.

36. Burnfield J. Normal and pathologic gait. In: Placzek JD, Boyce DA, editors. Orthopaedic physical therapy secrets. 3rd ed. St Louis, MO: Elsevier; 2016. p. 105-11.

37. Baker PN, Rushton S, Jameson SS, Reed M, Gregg P, Deehan DJ. Patient satisfaction with total knee replacement cannot be predicted from pre-operative variables alone: a cohort study from the National Joint Registry for England and Wales. Bone Joint J 2013;95-B:1359-65.

38. Giesinger JM, Hamilton DF, Jost B, Behrend H, Giesinger K. WOMAC, EQ-5D and Knee Society Score Thresholds for Treatment Success After Total Knee Arthroplasty. J Arthroplasty 2015;30:2154-8.

39. Dobson F, Hinman RS, Roos EM, Abbott JH, Stratford P, Davis AM, et al. OARSI recommended performance-based tests to assess physical function in people diagnosed with hip or knee osteoarthritis. Osteoarthritis Cartilage 2013;21:1042-52.

40. Webster KE, Wittwer JE, Feller JA. Quantitative gait analysis after medial unicompartmental knee arthroplasty for osteoarthritis. J Arthroplasty 2003;18:751-9.

41. McClelland JA, Webster KE, Feller JA, Menz HB. Knee kinematics during walking at different speeds in people who have undergone total knee replacement. Knee 2011;18:151-5.

42. Suh MJ, Kim BR, Kim SR, Han EY, Nam KW, Lee SY, et al. Bilateral quadriceps muscle strength and pain correlate with gait speed and gait endurance early after unilateral total knee arthroplasty: a cross-sectional study. Am J Phys Med Rehabil 2019; 98:897-905. 